\title{
Ointment Dosage Form Category
}

National Cancer Institute

\section{Source}

National Cancer Institute. Ointment Dosage Form Category. NCI Thesaurus. Code C154557.

A type of semi-solid pharmaceutical dose form consisting of a single-phase basis in which solids or liquids may be dispersed; ointments may be composed of a variety of bases including hydrocarbons, emulsifiers, vegetable oils and animal fats; they may be hydrophobic (bases made from substances such as hard, liquid, or light liquid paraffins, vegetable oils, animal fats, synthetic glycerides, waxes and liquid polyalkylsiloxanes, and which are only able to absorb a small amount of water), hydrophilic (with a water-miscible basis, usually a mixture of liquid and solid macrogols) or water-emulsifying (capable of absorbing larger amounts of water and forming emulsions, with bases containing waterin-oil emulsifiers such as wool alcohols, sorbitan esters, monog lycerides and fatty alcohols, or oil-in-water emulsifiers such as sulfated fatty alcohols, polysorbates, macrogol cetostearyl ether, esters of fatty acids with macrogols). 